\title{
Dunkl-spherical maximal function
}

\author{
Abdessattar Jemai \\ January 23, 2018
}

\begin{abstract}
In this paper, we study the $L^{p}$-boundedness of the spherical maximal function associated to the Dunkl operators.
\end{abstract}

Keywords: Dunkl operators; Dunkl transform; Dunkl translations; Spherical maximal function.

2010 AMS Mathematics Subject Classification: 42B10, 42B25, 44A15, $44 \mathrm{~A} 35$.

\section{Introduction and backgrounds}

In [13], E. Stein introduced the spherical maximal function by

$$
M(f)(x)=\sup _{r>0} \int_{\mathbb{S}^{d-1}} f(x-r y) d \sigma(y)
$$

where $d \sigma$ is the surface measure on $\mathbb{S}^{d-1}$ and showed that $M$ is bounded from $L^{p}\left(\mathbb{R}^{d}\right)$ to $L^{p}\left(\mathbb{R}^{d}\right)$ for the optimal range $p>\frac{d}{d-1}$ with $d \geq 3$. The case $d=2$ was proved by Bourgain in [1]. The aim of this work is to extend these results to the Dunkl setting.

To begin, we recall some results in Dunkl theory (see [3, 4, 6, 17, 9, 15]) and we refer for more details to the survey [8].

Let $G \subset O\left(\mathbb{R}^{d}\right)$ be a finite reflection group associated to a reduced root system $R$. For $\alpha \in R$, we denote by $\mathbb{H}_{\alpha}$ the hyperplane orthogonal to $\alpha$. We denote by $k$ a nonnegative multiplicity function defined on $R$ with the property that $k$ is $G$-invariant. For a given $\beta \in \mathbb{R}^{d} \backslash \bigcup_{\alpha \in R} \mathbb{H}_{\alpha}$, we fix a 
positive subsystem $R_{+}=\{\alpha \in R:\langle\alpha, \beta\rangle>0\}$. We associate with $k$ the index $\gamma=\sum_{\xi \in R_{+}} k(\xi)$ and a weighted measure $\nu_{k}$ given by

$$
d \nu_{k}(x):=w_{k}(x) d x \quad \text { where } \quad w_{k}(x)=\prod_{\xi \in R_{+}}|\langle\xi, x\rangle|^{2 k(\xi)}, \quad x \in \mathbb{R}^{d} .
$$

Further, we introduce the Mehta-type constant $c_{k}$ by

$$
c_{k}=\left(\int_{\mathbb{R}^{d}} e^{-\frac{\|x\|^{2}}{2}} w_{k}(x) d x\right)^{-1} .
$$

For every $1 \leq p \leq+\infty$, we denote by $L_{k}^{p}\left(\mathbb{R}^{d}\right)$, the spaces $L^{p}\left(\mathbb{R}^{d}, d \nu_{k}(x)\right)$, and we use \|\|$_{p, k}$ as a shorthand for \|\|$_{L_{k}^{p}\left(\mathbb{R}^{d}\right)}$.

By using the homogeneity of degree $2 \gamma$ of $w_{k}$, for a radial function $f$ in $L_{k}^{1}\left(\mathbb{R}^{d}\right)$, there exists a function $F$ on $[0,+\infty)$ such that $f(x)=F(\|x\|)$, for all $x \in \mathbb{R}^{d}$. The function $F$ is integrable with respect to the measure $r^{2 \gamma+d-1} d r$ on $[0,+\infty)$ and we have

$$
\begin{aligned}
\int_{\mathbb{R}^{d}} f(x) d \nu_{k}(x) & =\int_{0}^{+\infty}\left(\int_{\mathbb{S}^{d-1}} f(r y) w_{k}(r y) d \sigma(y)\right) r^{d-1} d r \\
& =\int_{0}^{+\infty}\left(\int_{\mathbb{S}^{d-1}} w_{k}(r y) d \sigma(y)\right) F(r) r^{d-1} d r \\
& =d_{k} \int_{0}^{+\infty} F(r) r^{2 \gamma+d-1} d r
\end{aligned}
$$

where $S^{d-1}$ is the unit sphere on $\mathbb{R}^{d}$ with the normalized surface measure $d \sigma$ and

$$
d_{k}=\int_{\mathbb{S}^{d-1}} w_{k}(x) d \sigma(x)=\frac{c_{k}^{-1}}{2^{\gamma+\frac{d}{2}-1} \Gamma\left(\gamma+\frac{d}{2}\right)} .
$$

The Dunkl operators $T_{j}, 1 \leq j \leq d$ are the following $k$-deformations of directional derivatives $\frac{\partial}{x_{j}}$ given by:

$$
T_{j} f(x)=\frac{\partial f}{\partial x_{j}}(x)+\sum_{\alpha \in R_{+}} k(\alpha) \alpha_{j} \frac{f(x)-f\left(\rho_{\alpha}(x)\right)}{\langle\alpha, x\rangle}, \quad f \in \mathcal{E}\left(\mathbb{R}^{d}\right), \quad x \in \mathbb{R}^{d},
$$

where $\rho_{\alpha}$ is the reflection on the hyperplane $\mathbb{H}_{\alpha}$ and $\alpha_{j}=\left\langle\alpha, e_{j}\right\rangle,\left(e_{1}, \ldots, e_{d}\right)$ being the canonical basis of $\mathbb{R}^{d}$. 
Notice that in the case $k \equiv 0$, the weighted function $w_{k} \equiv 1$, the measure $\nu_{k}$ coincide with the Lebesgue measure and the operator $T_{\xi}$ reduced to the corresponding partial derivatives $\frac{\partial}{x_{j}}$. Therefore Dunkl analysis can be viewed as a generalization of classical Fourier analysis.

For $y \in \mathbb{C}^{d}$, the system

$$
\left\{\begin{array}{l}
T_{j} u(x, y)=y_{j} u(x, y), \quad 1 \leq j \leq d \\
u(0, y)=1
\end{array}\right.
$$

admits a unique analytic solution on $\mathbb{R}^{d}$, denoted by $E_{k}(x, y)$ and called the Dunkl kernel. This kernel has a unique holomorphic extension to $\mathbb{C}^{d} \times \mathbb{C}^{d}$. We have for all $\lambda \in \mathbb{C}$ and $z, z^{\prime} \in \mathbb{C}^{d}, E_{k}\left(z, z^{\prime}\right)=E_{k}\left(z^{\prime}, z\right), E_{k}\left(\lambda z, z^{\prime}\right)=$ $E_{k}\left(z, \lambda z^{\prime}\right)$ and for $x, y \in \mathbb{R}^{d},\left|E_{k}(x, i y)\right| \leq 1$.

The Dunkl transform $\mathcal{F}_{k}$ is defined for $f \in \mathcal{D}\left(\mathbb{R}^{d}\right)$ by

$$
\mathcal{F}_{k}(f)(x)=c_{k} \int_{\mathbb{R}^{d}} f(y) E_{k}(-i x, y) d \nu_{k}(y), \quad x \in \mathbb{R}^{d}
$$

We list some known properties of this transform:

i) The Dunkl transform of a function $f \in L_{k}^{1}\left(\mathbb{R}^{d}\right)$ has the following basic property

$$
\left\|\mathcal{F}_{k}(f)\right\|_{\infty, k} \leq\|f\|_{1, k}
$$

ii) The Dunkl transform is an automorphism on the Schwartz space $\mathcal{S}\left(\mathbb{R}^{d}\right)$.

iii) When both $f$ and $\mathcal{F}_{k}(f)$ are in $L_{k}^{1}\left(\mathbb{R}^{d}\right)$, we have the inversion formula

$$
f(x)=\int_{\mathbb{R}^{d}} \mathcal{F}_{k}(f)(y) E_{k}(i x, y) d \nu_{k}(y), \quad x \in \mathbb{R}^{d} .
$$

iv) (Plancherel's theorem) The Dunkl transform on $\mathcal{S}\left(\mathbb{R}^{d}\right)$ extends uniquely to an isometric automorphism on $L_{k}^{2}\left(\mathbb{R}^{d}\right)$.

The Dunkl translation operators $\tau_{x}, x \in \mathbb{R}^{d}$ is defined on $L_{k}^{2}\left(\mathbb{R}^{d}\right)$ by

$$
\mathcal{F}_{k}\left(\tau_{x}(f)\right)(y)=E_{k}(i x, y) \mathcal{F}_{k}(f)(y) \quad y \in \mathbb{R}^{d} .
$$


As an operator on $L_{k}^{2}\left(\mathbb{R}^{d}\right), \tau_{x}$ is bounded. According to ([15], Theorem 3.7), the operator $\tau_{x}$ can be extended to the space of radial functions $L_{k}^{p}\left(\mathbb{R}^{d}\right)^{r a d}, 1 \leq p \leq 2$ and we have for a function $f$ in $L_{k}^{p}\left(\mathbb{R}^{d}\right)^{r a d}$,

$$
\left\|\tau_{x}(f)\right\|_{p, k} \leq\|f\|_{p, k} .
$$

It was shown in [10] that if $f$ is a radial function in $\mathcal{S}\left(\mathbb{R}^{d}\right)$ with $f(y)=\widetilde{f}(\|y\|)$, then

$$
\tau_{x}(f)(y)=\int_{\mathbb{R}^{d}} \tilde{f}(A(x, y, \eta)) d \mu_{x}(\eta)
$$

where $A(x, y, \eta)=\sqrt{\|x\|^{2}+\|y\|^{2}-2<y, \eta>}$ and $\mu_{x}$ is a probability measure supported in the convex hull $c o(G . x)$ of the $G$-orbit of $x$ in $\mathbb{R}^{d}$. We observe that,

$$
\eta \in c o(G \cdot x) \Longrightarrow \min _{g \in G}\|g \cdot x-y\| \leq A(x, y, \eta) \leq \max _{g \in G}\|g \cdot x-y\| .
$$

We collect below some useful facts:

i) For all $x, y \in \mathbb{R}^{d}, \tau_{x}(f)(y)=\tau_{y}(f)(x)$.

ii) For $f \in L_{k}^{2}\left(\mathbb{R}^{d}\right) \cap L_{k}^{1}\left(\mathbb{R}^{d}\right)$

$$
\int_{\mathbb{R}^{d}} \tau_{x}(f)(y) d \nu_{k}(y)=\int_{\mathbb{R}^{d}} f(y) d \nu_{k}(y) .
$$

iii) For all $x \in \mathbb{R}^{d}$ and $f, g \in L_{k}^{2}\left(\mathbb{R}^{d}\right)$

$$
\int_{\mathbb{R}^{d}} \tau_{x}(f)(y) g(y) d \nu_{k}(y)=\int_{\mathbb{R}^{d}} f(y) \tau_{x}(g)(y) d \nu_{k}(y) .
$$

The Dunkl convolution product $*_{k}$ of two functions $f$ and $g$ in $L_{k}^{2}\left(\mathbb{R}^{d}\right)$ is given by

$$
\left(f *_{k} g\right)(x)=\int_{\mathbb{R}^{d}} \tau_{x}(f)(-y) g(y) d \nu_{k}(y), \quad x \in \mathbb{R}^{d} .
$$

The Dunkl convolution product is commutative and for $f, g \in \mathcal{D}\left(\mathbb{R}^{d}\right)$, we have

$$
\mathcal{F}_{k}\left(f *_{k} g\right)=\mathcal{F}_{k}(f) \mathcal{F}_{k}(g)
$$


It was proved in ([15], Theorem 4.1) that when $g$ is a bounded radial function in $L_{k}^{1}\left(\mathbb{R}^{d}\right)$, then the application $f \longrightarrow f *_{k} g$ initially defined on the intersection of $L_{k}^{1}\left(\mathbb{R}^{d}\right)$ and $L_{k}^{2}\left(\mathbb{R}^{d}\right)$ extends to $L_{k}^{p}\left(\mathbb{R}^{d}\right), 1 \leq p \leq+\infty$ as a bounded operator. In particular,

$$
\left\|f *_{k} g\right\|_{p, k} \leq\|f\|_{p, k}\|g\|_{1, k} .
$$

(see [10, 11] ) The Dunkl transform of $\sigma$ is given by

$$
\mathcal{F}_{k}(\sigma)(x)=\frac{1}{c_{k}} \int_{\mathbb{S}^{d-1}} E_{k}(-i x, y) \omega_{k}(y) d \sigma(y)=c_{\gamma} j_{\frac{2 \gamma+d}{2}-1}(\|x\|),
$$

where $j_{\frac{2 \gamma+d}{2}-1}$ is the Bessel function of the first type and $c_{\gamma}=\frac{1}{2^{\gamma+\frac{d}{2}-1} \Gamma\left(\gamma+\frac{d}{2}\right)}$. In particular (see [14]), the function

$$
\begin{gathered}
\mathcal{F}_{k}(\sigma)(r \xi)=O\left(r^{-\frac{2 \gamma+d-1}{2}}\right), \quad r \rightarrow+\infty . \\
\frac{1}{r} \frac{\partial}{\partial r} \mathcal{F}_{k}(\sigma)(r \xi)=O\left(r^{-\frac{2 \gamma+d+1}{2}}\right), \quad r \rightarrow+\infty .
\end{gathered}
$$

Along this paper we use $C$ to denote a suitable positive constant which is not necessarily the same in each occurrence and we write for $x \in \mathbb{R}^{d},\|x\|=$ $\sqrt{\langle x, x\rangle}$. Furthermore, we denote by

- $\mathcal{E}\left(\mathbb{R}^{d}\right)$ the space of infinitely differentiable functions on $\mathbb{R}^{d}$.

- $\mathcal{S}\left(\mathbb{R}^{d}\right)$ the Schwartz space of functions in $\mathcal{E}\left(\mathbb{R}^{d}\right)$ which are rapidly decreasing as well as their derivatives.

- $\mathcal{D}\left(\mathbb{R}^{d}\right)$ the subspace of $\mathcal{E}\left(\mathbb{R}^{d}\right)$ of compactly supported functions.

\section{Dunkl-spherical maximal function}

For $f \in L_{k}^{2}\left(\mathbb{R}^{d}\right)$, we define the maximal function $\mathcal{M}_{k} f$ by

$$
\mathcal{M}_{k}(f)(x)=\sup _{r>0} \frac{1}{\nu_{k}(B(0, r))}\left|\int_{B(0, r)} \tau_{x} f(y) d \nu_{k}(y)\right|,
$$

where $B(0, r)$ is the ball of radius $r$ centered at 0 and $x \in \mathbb{R}^{d}$.

It was proved in [15] that the maximal function is bounded on $f \in L_{k}^{p}\left(\mathbb{R}^{d}\right)$ for $1<p \leq \infty$, and of weak type $(1,1)$, for $f \in L_{k}^{1}\left(\mathbb{R}^{d}\right)$ that is $a>0$,

$$
\int_{E(a)} d \nu_{k}(x) \leq \frac{C}{a}\|f\|_{1, k}
$$


where $E(a)=\left\{x: \mathcal{M}_{k} f(x)>a\right\}$ and $C$ is a constant independent of $a$ and $f$.

As in [5], we define the spherical mean operator on $A_{k}\left(\mathbb{R}^{d}\right)=\left\{f \in L_{k}^{1}\left(\mathbb{R}^{d}\right): \mathcal{F}_{k}(f) \in L_{k}^{1}\left(\mathbb{R}^{d}\right)\right\}$, for $x \in \mathbb{R}^{d}$ and $r>0$ by

$$
\begin{aligned}
\mathbf{S}_{r}(f)(x) & =\frac{1}{d_{k}} \int_{\mathbb{S}^{d-1}} \tau_{x}(f(-r y)) \omega_{k}(y) d \sigma(y) \\
& =\frac{1}{d_{k}} \int_{r \mathbb{S}^{d-1}} \tau_{x}(f(-y)) \omega_{k}\left(\frac{y}{r}\right) d \sigma_{r}(y)
\end{aligned}
$$

Now, the Dunkl-spherical maximal function $M(f)$ is given by

$$
M(f)(x)=\sup _{r>0}\left|\mathbf{S}_{r}(f)(x)\right|, \quad x \in \mathbb{R}^{d} .
$$

Theorem 2.1 Let $2 \gamma+d \geq 2$ and $\frac{2 \gamma+d}{2 \gamma+d-1}<p<2 \gamma+d$. Then there exists a constant $C>0$ such that for all $f \in L_{k}^{p}\left(\mathbb{R}^{d}\right)$,

$$
\|M(f)\|_{p, k} \leq C\|f\|_{p, k}
$$

Before proving the theorem, we need to establish some useful results. In fact, fix a function $\psi_{0} \in \mathcal{S}\left(\mathbb{R}^{d}\right)$ which is the Dunkl transform of a $\mathcal{C}^{\infty}$-radial function with compact support such that:

$$
\psi_{0}(0)=1, \quad\left(\frac{\partial^{i}}{\partial r^{i}} \psi_{0}\right)(0)=0
$$

for $1 \leq i<\frac{2 \gamma+d}{2}$ and where $\frac{\partial}{\partial r}$ denotes the derivation in the radial direction. To obtain a such function, taking $\psi \in \mathcal{S}\left(\mathbb{R}^{d}\right)$ with $\mathcal{F}_{k}(\psi)$ is a $\mathcal{C}^{\infty}$ - radial function with compact support and

$$
\psi(0) \neq 0 \quad \text { and } \quad \psi_{0}(r \xi)=\left(\sum_{j=0}^{\left[\frac{2 \gamma+d}{2}\right]} a_{j} r^{j}\right) \psi(r \xi)
$$

for $\xi \in \mathbb{S}^{d-1}$. The coefficients $a_{j}$ are solutions of triangular system given by the conditions (2.4) and $\left[\frac{2 \gamma+d}{2}\right]$ is the least integer not less than $\frac{2 \gamma+d}{2}$.

Now set the functions $\psi_{j}$ with

$$
\psi_{1}(y)=\psi_{0}\left(\frac{y}{2}\right)-\psi_{0}(y) \quad \text { and } \quad \psi_{j}(y)=\psi_{1}\left(2^{-(j-1)} y\right), \quad j \geq 1 .
$$


Thus, $\psi_{j}$ is radial and $\mathcal{F}_{k}\left(\psi_{j}\right)$ is a compact supported functions. It follows that for some constants $t, C>0$ we have

$$
\left|\psi_{1}(y)\right| \leq C\|y\|^{\frac{2 \gamma+d}{2}}, \quad \text { if } \quad\|y\| \leq t
$$

and

$$
\sum_{j=0}^{+\infty} \psi_{j}(y)=1, \quad y \in \mathbb{R}^{d}
$$

Let $m_{j}=\mathcal{F}_{k}(\sigma) \psi_{j}$ and let $\varphi_{j}$ the function in $\mathcal{S}\left(\mathbb{R}^{d}\right)$ such that $\mathcal{F}_{k}\left(\varphi_{j}\right)=m_{j}$. It follows that for $f \in \mathcal{S}\left(\mathbb{R}^{d}\right)$,

$$
\mathbf{S}_{r}(f)(x)=\sum_{j=0}^{+\infty} f *_{k} \varphi_{j}
$$

In fact, this can be done because from (2.7), we have

$$
\mathcal{F}_{k}(\sigma)(y)=\sum_{j=0}^{+\infty} \mathcal{F}_{k}(\sigma)(y) \psi_{j}(y)=\sum_{j=0}^{+\infty} \mathcal{F}_{k}\left(\varphi_{j}\right)(y)
$$

and we can write

$$
\mathcal{F}_{k}\left(\mathbf{S}_{r}(f)\right)(y)=\sum_{j=0}^{+\infty} \mathcal{F}_{k}\left(f *_{k} \varphi_{j}\right)(y)
$$

which implies with the inversion formula that

$\mathbf{S}_{r}(f)(y)=\mathcal{F}_{k}^{-1}\left(\sum_{j=0}^{+\infty} \mathcal{F}_{k}\left(f *_{k} \varphi_{j}\right)\right)(y)=\int_{\mathbb{R}^{d}} \sum_{j=0}^{+\infty} \mathcal{F}_{k}\left(f *_{k} \varphi_{j}\right)(z) E_{k}(i y, z) d \nu_{k}(z)$.

To interchange the sum and the integral, we proceed as follows:

From (1.5), we have 


$$
\begin{aligned}
& \sum_{j=1}^{+\infty}\left(\int_{\mathbb{R}^{d}}\left|\mathcal{F}_{k}\left(f *_{k} \varphi_{j}\right)(z) E_{k}(i y, z)\right| d \nu_{k}(z)\right) \\
& \quad \leq \sum_{j=1}^{+\infty}\left(\int_{\mathbb{R}^{d}}\left|\mathcal{F}_{k}\left(\varphi_{j}\right)(z) \| \mathcal{F}_{k}(f)(z)\right| d \nu_{k}(z)\right) \\
& \quad \leq \sum_{j=1}^{+\infty}\left(\int_{\mathbb{R}^{d}}\left|\psi_{j}(z) \| \mathcal{F}_{k}(f)(z)\right| d \nu_{k}(z)\right) \\
& \quad \leq \sum_{j=1}^{+\infty}\left(\int_{\|z\| \leq 2^{j-1} t}\left|\psi_{j}(z)\left\|\mathcal{F}_{k}(f)(z)\left|d \nu_{k}(z)+\int_{\|z\| \geq 2^{j-1} t}\right| \psi_{j}(z)\right\| \mathcal{F}_{k}(f)(z)\right| d \nu_{k}(z)\right)
\end{aligned}
$$

By using (2.5) and (2.6), we obtain

$$
\begin{aligned}
\int_{\|y\| \leq 2^{j-1} t}\left|\psi_{j}(z) \| \mathcal{F}_{k}(f)(z)\right| d \nu_{k}(z) & \leq c 2^{-(j-1) \frac{2 \gamma+d}{2}} \int_{\|y\| \leq 2^{j-1} t}\|z\|^{\frac{2 \gamma+d}{2}}\left|\mathcal{F}_{k}(f)(z)\right| d \nu_{k}(z) \\
& \leq c 2^{-(j-1) \frac{2 \gamma+d}{2}} \int_{\mathbb{R}^{d}}\|z\|^{\frac{2 \gamma+d}{2}}\left|\mathcal{F}_{k}(f)(z)\right| d \nu_{k}(z)
\end{aligned}
$$

and

$$
\begin{aligned}
\int_{\|z\| \geq 2^{j-1} t}\left|\psi_{j}(z) \| \mathcal{F}_{k}(f)(z)\right| d \nu_{k}(z) & =\int_{\frac{\|z\|}{2^{j-1}} \geq 1}\left|\psi_{j}(z) \| \mathcal{F}_{k}(f)(z)\right| d \nu_{k}(z) \\
& \leq 2^{-(j-1)} t \int_{\mathbb{R}^{d}}\|z\|\left|\psi_{j}(z) \| \mathcal{F}_{k}(f)(z)\right| d \nu_{k}(z) \\
& \leq 2^{-(j-1)} t \int_{\mathbb{R}^{d}}\|z\|\left|\mathcal{F}_{k}(f)(z)\right| d \nu_{k}(z)
\end{aligned}
$$

therefore $\sum_{j=1}^{+\infty}\left(\int_{\mathbb{R}^{d}}\left|\mathcal{F}_{k}\left(f *_{k} \varphi_{j}\right)(z) E_{k}(i y, z)\right| d \nu_{k}(z)\right)$ converge.

For all $j \geq 0$ and $r>0$, we define the function $\varphi_{j, r}(x)=r^{-2 \gamma-d} \varphi_{j}\left(\frac{x}{r}\right)$. Then we can write,

$$
\mathbf{S}_{r}(f)(x)=\sum_{j=0}^{+\infty} f *_{k} \varphi_{j, r}
$$

Hence for $f \in \mathcal{S}\left(\mathbb{R}^{d}\right)$, we obtain

$$
M(f) \leq \sum_{j=0}^{+\infty} M_{\varphi_{j}}(f)
$$


where $M_{\varphi_{j}}(f)(x)=\sup _{r>0}\left|f *_{k} \varphi_{j, r}(x)\right|=\sup _{r>0}\left|\int_{\mathbb{R}^{d}} \tau_{x}(f)(y) \varphi_{j, r}(y) d \nu_{k}(y)\right|$.

To prove the theorem, it suffices to establish an inequality of the form

$$
\left\|M_{\varphi_{j}}(f)\right\|_{p, k} \leq C_{j, p}\|f\|_{p, k}
$$

with $\sum_{j=0}^{+\infty} C_{j, p}<+\infty$

Lemma 2.1 There exists a constant $C>0$ such that, for any $x \in \mathbb{R}^{d}$ and $j \geq 0$

$$
\left|\varphi_{j}(x)\right| \leq C \frac{2^{j}}{(1+\|x\|)^{2 \gamma+d+1}}
$$

Proof. 2.1 Remember that $\varphi_{j}=\mathbf{S}_{r}\left(\mathcal{F}_{k}^{-1}\left(\psi_{j}\right)\right)$ for $j \geq 1$. We have,

$$
\mathcal{F}_{k}^{-1}\left(\psi_{j}\right)(x)=2^{(j-1)(2 \gamma+d)} \mathcal{F}_{k}^{-1}\left(\psi_{1}\right)\left(2^{j-1} x\right) .
$$

Since $\mathcal{F}_{k}^{-1}\left(\psi_{0}\right)$ and $\mathcal{F}_{k}^{-1}\left(\psi_{1}\right)$ are in $\mathcal{S}\left(\mathbb{R}^{d}\right)$, then

$$
\left|\mathcal{F}_{k}^{-1}\left(\psi_{0}\right)(x)\right| \text { and }\left|\mathcal{F}_{k}^{-1}\left(\psi_{1}\right)(x)\right| \text { are bounded by } \frac{C}{(1+\|x\|)^{2 \gamma+d+1}} .
$$

Taking $\phi_{j}(x, y, \xi)=\mathcal{F}_{k}^{-1}\left(\psi_{1}\right)\left(2^{j-1} \sqrt{\|x\|^{2}+\|y\|^{2}-2<y, \xi>}\right)$ for $j \geq 1$ and using (1.1), we get

$$
\begin{aligned}
\left|\varphi_{j}(x)\right| & =\left|\int_{\mathbb{S}^{d-1}} \tau_{x}\left(\mathcal{F}_{k}^{-1}\left(\psi_{j}\right)\right)(-y) \omega_{k}(y) d \sigma(y)\right| \\
& \leq 2^{(j-1)(2 \gamma+d)} \int_{\mathbb{S}^{d-1}}\left(\int_{\mathbb{R}^{d}}\left|\phi_{j}(x, y, \xi)\right| d \mu_{x}(\xi)\right) \omega_{k}(y) d \sigma(y) .
\end{aligned}
$$

For $j=0$, we have by (1.1)

$$
\begin{aligned}
\left|\varphi_{0}(x)\right| & =\left|\int_{\mathbb{S}^{d-1}} \tau_{x}\left(\mathcal{F}_{k}^{-1}\left(\psi_{0}\right)\right)(-y) \omega_{k}(y) d \sigma(y)\right| \\
& \leq \int_{\mathbb{S}^{d-1}}\left(\int_{\mathbb{R}^{d}}\left|\mathcal{F}_{k}^{-1}\left(\psi_{0}\right)\left(\sqrt{\|x\|^{2}+\|y\|^{2}-2<y, \xi>}\right)\right| d \mu_{x}(\xi)\right) \omega_{k}(y) d \sigma(y) .
\end{aligned}
$$


From (1.2), (2.10), (2.11) and (2.12), we get for $j \geq 0$ $\left|\varphi_{j}(x)\right|$

$$
\begin{aligned}
& \leq C 2^{j(2 \gamma+d)} \int_{\mathbb{S}^{d-1}}\left(\int_{\mathbb{R}^{d}} \frac{1}{\left(1+2^{j} \min _{g \in G}\|g x-y\|\right)^{2 \gamma+d+1}} d \mu_{x}(\xi)\right) \omega_{k}(y) d \sigma(y) \\
& \leq C 2^{j(2 \gamma+d)} \int_{\mathbb{S}^{d-1}} \frac{1}{\left(1+2^{j} \min _{g \in G}\|g x-y\|\right)^{2 \gamma+d+1}} \omega_{k}(y) d \sigma(y) .
\end{aligned}
$$

If $\|x\|>2$, then $\|g x-y\| \geq\|x\|-1 \geq \frac{\|x\|}{2}$ and we have

$$
\begin{aligned}
\left|\varphi_{j}(x)\right| & \leq \frac{C 2^{j(2 \gamma+d)}}{\left(1+2^{j-1}\|x\|\right)^{2 \gamma+d+1}} \\
& \leq \frac{C 2^{-j}}{\|x\|^{2 \gamma+d+1}} \\
& \leq C \frac{2^{j}}{(1+\|x\|)^{2 \gamma+d+1}} .
\end{aligned}
$$

If $\|x\| \leq 2$ then,

$$
\begin{aligned}
\left|\varphi_{j}(x)\right| & \leq \int_{\mathbb{S}^{d-1}} \frac{2^{j(2 \gamma+d)}}{\left(1+2^{j} \min _{g \in G}\|g x-y\|\right)^{2 \gamma+d+1}} \omega_{k}(y) d \sigma(y) \\
& \leq 2^{j(2 \gamma+d)} \int_{\left\{y \in \mathbb{S}^{d-1} ; \min _{g \in G}\|g x-y\| \leq 2^{-j}\right\}} \omega_{k}(y) d \sigma(y) \\
& +2^{j(2 \gamma+d)} \sum_{i=0}^{+\infty} 2^{-(2 \gamma+d+1) i} \int_{\left\{y \in \mathbb{S}^{d-1} ; \min _{g \in G}\|g x-y\| \leq 2^{i+1-j}\right\}} \omega_{k}(y) d \sigma(y) \\
& \leq C\left(2^{j}+2^{j} \sum_{i=0}^{+\infty} 2^{-2 i}\right) \leq C \frac{2^{j}}{(1+\|x\|)^{2 \gamma+d+1}} .
\end{aligned}
$$

From (2.13) and 2.14), we obtain

$$
\left|\varphi_{j}(x)\right| \leq C \frac{2^{j}}{(1+\|x\|)^{2 \gamma+d+1}}
$$

Lemma 2.2 There exists a constant $C>0$ such that, for any $f \in \mathcal{S}\left(\mathbb{R}^{d}\right)$, $j \geq 0$ and $\alpha>0$,

$$
\int_{\widetilde{E}(\alpha)} d \nu_{k}(x) \leq \frac{C 2^{j}}{\alpha}\|f\|_{1, k} .
$$


where $\widetilde{E}(\alpha)=\left\{x \in \mathbb{R}^{d} ; M_{\varphi_{j}}(f)(x)>\alpha\right\}$ and $C$ is a constant depending only on $d$.

Proof. 2.2 Let first prove that, for $f \in \mathcal{S}\left(\mathbb{R}^{d}\right)$ and $x \in \mathbb{R}^{d}$,

$$
\int_{\mathbb{R}^{d}} \tau_{x}\left(\left|\varphi_{j, r}\right|\right)(y)|f(y)| d \nu_{k}(y) \leq C 2^{j} \mathcal{M}_{k}(|f|)(x) \quad j \geq 0,
$$

where $\mathcal{M}_{k}(f)$ is given by (2.1).

We denote by $A_{i}=\left\{y \in \mathbb{R}^{d}, r 2^{i} \leq\|y\|<r 2^{i+1}\right\}$, then

$\tau_{x}\left(\left|\varphi_{j, r}\right|\right)(y)=\tau_{x}\left(\sum_{i=-\infty}^{+\infty}\left|\varphi_{j, r}\right| \cdot \chi_{A_{i}}\right)(y)=\sum_{i=-\infty}^{+\infty} \tau_{x}\left(\left|\varphi_{j, r}\right| \cdot \chi_{A_{i}}\right)(y)$.

From (2.9), one has

$$
\begin{aligned}
\left(\left|\varphi_{j, r}\right| \cdot \chi_{A_{i}}\right)(y) & \leq C 2^{j} \frac{r^{-(2 \gamma+d)}}{\left(1+\frac{\|y\|}{r}\right)^{2 \gamma+d+1}} \chi_{A_{i}}(y) \\
& \leq C 2^{j} \frac{r^{-(2 \gamma+d)}}{\left(1+2^{i}\right)^{2 \gamma+d+1}} \chi_{A_{i}}(y)
\end{aligned}
$$

and since $\left|\varphi_{j, r}\right| \cdot \chi_{A_{i}}$ is a radial function, this implies that

$$
\tau_{x}\left(\left|\varphi_{j, r}\right| \cdot \chi_{A_{i}}\right)(y) \leq C 2^{j} \frac{r^{-(2 \gamma+d)}}{\left(1+2^{i}\right)^{2 \gamma+d+1}} \tau_{x}\left(\chi_{A_{i}}\right)(y) .
$$

Using (1.4), we obtain

$$
\begin{aligned}
& \int_{\mathbb{R}^{d}} \tau_{x}\left(\left|\varphi_{j, r}\right|\right)(y)|f(y)| d \nu_{k}(y) \\
& \leq C 2^{j} \int_{\mathbb{R}^{d}} \sum_{i=-\infty}^{+\infty} \frac{r^{-(2 \gamma+d)}}{\left(1+2^{i}\right)^{2 \gamma+d+1}} \tau_{x}\left(\chi_{A_{i}}\right)(y)|f(y)| d \nu_{k}(y) \\
& \leq C 2^{j} \sum_{i=-\infty}^{+\infty} \frac{r^{-(2 \gamma+d)}}{\left(1+2^{i}\right)^{2 \gamma+d+1}} \int_{\mathbb{R}^{d}} \chi_{A_{i}}(y) \tau_{x}(|f|)(y) d \nu_{k}(y) \\
& \leq C 2^{j} \sum_{i=-\infty}^{+\infty} \frac{r^{-(2 \gamma+d)}}{\left(1+2^{i}\right)^{2 \gamma+d+1}} \int_{B\left(0, r 2^{i+1}\right)} \tau_{x}(|f|)(y) d \nu_{k}(y) \\
& \leq C 2^{j} \sum_{i=-\infty}^{+\infty} \frac{r^{-(2 \gamma+d)}}{\left(1+2^{i}\right)^{2 \gamma+d+1}}\left(r 2^{i+1}\right)^{2 \gamma+d} \mathcal{M}_{k}(|f|)(x) \\
& \leq C 2^{j} \mathcal{M}_{k}(|f|)(x) .
\end{aligned}
$$


By the fact that,

$$
\begin{aligned}
\left|\int_{\mathbb{R}^{d}} \tau_{x}\left(\varphi_{j, r}\right)(y) f(y) d \nu_{k}(y)\right| & \leq \int_{\mathbb{R}^{d}}\left|\tau_{x}\left(\varphi_{j, r}\right)(y)\right||f(y)| d \nu_{k}(y) \\
& \leq \int_{\mathbb{R}^{d}} \tau_{x}\left(\left|\varphi_{j, r}\right|\right)(y)|f(y)| d \nu_{k}(y)
\end{aligned}
$$

we deduce

$$
M_{\varphi_{j}}(f)(x) \leq C 2^{j} \mathcal{M}_{k}(|f|)(x) .
$$

From (2.2), we conclude the proof of the lemma.

Lemma 2.3 There exists a constant $C>0$ such that, for any $f \in \mathcal{S}\left(\mathbb{R}^{d}\right)$, $j \geq 1$,

$$
\left\|M_{\varphi_{j}}(f)\right\|_{2, k} \leq C 2^{-j \frac{2 \gamma+d-2}{2}}\|f\|_{2, k} .
$$

Proof. 2.3 (See [13], [16]) For $f \in \mathcal{S}\left(\mathbb{R}^{d}\right)$, we have from (1.5)

$$
\mathcal{F}_{k}\left(f *_{k} \varphi_{j, r}\right)(x)=\mathcal{F}_{k}(f)(x) \mathcal{F}_{k}\left(\varphi_{j, r}\right)(x)=\mathcal{F}_{k}(f)(x) m_{j}(r x) .
$$

Put

$$
g_{j}(f)(x)=\left(\int_{0}^{+\infty}\left|f *_{k} \varphi_{j, r}(x)\right|^{2} \frac{d r}{r}\right)^{\frac{1}{2}},
$$

the Littlewood-Paley function associated to the function $\varphi_{j, r}$. Using the Plancherel theorem and by (1.5), we obtain

$$
\begin{aligned}
\left\|g_{j}(f)\right\|_{2, k}^{2} & =\int_{\mathbb{R}^{d}}\left|g_{j}(f)(x)\right|^{2} d \nu_{k}(x) \\
& =\int_{\mathbb{R}^{d}}\left(\int_{0}^{+\infty}\left|f *_{k} \varphi_{j, r}(x)\right|^{2} \frac{d r}{r}\right) d \nu_{k}(x) \\
& =\int_{0}^{+\infty}\left(\int_{\mathbb{R}^{d}}\left|\mathcal{F}_{k}\left(f *_{k} \varphi_{j, r}\right)(x)\right|^{2} d \nu_{k}(x)\right) \frac{d r}{r} \\
& =\int_{0}^{+\infty}\left(\int_{\mathbb{R}^{d}}\left|\mathcal{F}_{k}(f)(x)\right|^{2}\left|m_{j}(r x)\right|^{2} d \nu_{k}(x)\right) \frac{d r}{r} \\
& \leq\|f\|_{2, k}^{2} \sup _{x \neq 0} \int_{0}^{+\infty}\left|m_{j}(r x)\right|^{2} \frac{d r}{r} .
\end{aligned}
$$


Since the function $m_{j}$ is radial, the integral is independent of $x$.

By using the definition of $m_{j}$, we have that

$$
\begin{aligned}
\int_{0}^{+\infty}\left|m_{j}(r x)\right|^{2} \frac{d r}{r} & =\int_{0}^{+\infty}\left|\mathcal{F}_{k}(\sigma)(r x)\right|^{2}\left|\psi_{j}(r x)\right|^{2} \frac{d r}{r} \\
& =\int_{0}^{+\infty}\left|\mathcal{F}_{k}(\sigma)(r x)\right|^{2}\left|\psi_{1}\left(2^{-j} r x\right)\right|^{2} \frac{d r}{r} \\
& =\int_{0}^{+\infty}\left|\mathcal{F}_{k}(\sigma)\left(2^{j} r x\right)\right|^{2}\left|\psi_{1}(r x)\right|^{2} \frac{d r}{r}
\end{aligned}
$$

From (1.6), 1.7) and (2.6), we obtain

$$
\begin{aligned}
\int_{0}^{+\infty}\left|m_{j}(r x)\right|^{2} \frac{d r}{r} & \leq C 2^{-j(2 \gamma+d-1)} \int_{0}^{+\infty} \frac{\left|\psi_{1}(r x)\right|^{2}}{r^{2 \gamma+d-1}} \frac{d r}{r} \\
& \leq C 2^{-j(2 \gamma+d-1)}
\end{aligned}
$$

this gives

$$
\left\|g_{j}(f)\right\|_{2, k} \leq C 2^{-\frac{j(2 \gamma+d-1)}{2}}\|f\|_{2} .
$$

Put now $\tilde{\varphi}_{j, r}(x)=r \frac{d}{d r} \varphi_{j, r}(x)$ and $\tilde{g}_{j}(f)$ the Littlewood-Paley function associated to $\tilde{\varphi}_{j, r}$, then

$$
\tilde{g}_{j}(f)(x)=\left(\int_{0}^{+\infty}\left|f *_{k} \tilde{\varphi}_{j, r}(x)\right|^{2} \frac{d r}{r}\right)^{\frac{1}{2}}=\left(\int_{0}^{+\infty} r\left|\frac{d}{d r} \varphi_{j, r} *_{k} f(x)\right|^{2} d r\right)^{\frac{1}{2}} .
$$

Similarly, with the use of (1.6) and (1.8), we have

$$
\begin{aligned}
\left\|\tilde{g}_{j}(f)\right\|_{2, k} & =\int_{\mathbb{R}^{d}}\left(\int_{0}^{+\infty} r\left|\frac{d}{d r} \varphi_{j, r} *_{k} f(x)\right|^{2} d r\right) d \nu_{k}(x) \\
& =C 2^{-j \frac{(2 \gamma+d-1)}{2}}\|f\|_{2, k} .
\end{aligned}
$$

Since $\lim _{r \rightarrow+\infty} f *_{k} \varphi_{j, r}(x)=0$, we have

$$
\begin{aligned}
\left|f *_{k} \varphi_{j, r}(x)\right|^{2} & =-2 \operatorname{Re}\left(\int_{r}^{+\infty} \overline{\varphi_{j, s} *_{k} f(x)} \frac{d}{d s} \varphi_{j, s} *_{k} f(x) d s\right) \\
& =-2 \operatorname{Re}\left(\int_{r}^{+\infty} \overline{\varphi_{j, s} *_{k} f(x)} \tilde{\varphi}_{j, s} *_{k} f(x) \frac{d s}{s}\right) \\
& \leq 2 \int_{r}^{+\infty}\left|\overline{\varphi_{j, s} *_{k} f(x)}\right|\left|\tilde{\varphi}_{j, s} *_{k} f(x)\right| \frac{d s}{s} .
\end{aligned}
$$


Using Cauchy-Schwartz's inequality, we deduce that

$$
\sup _{r>0}\left|f *_{k} \varphi_{j, r}(x)\right|^{2} \leq 2 g_{j}(f)(x) \tilde{g}_{j}(f)(x) .
$$

Integrating over $\mathbb{R}^{d}$ and using again the Cauchy-Schwartz inequality, we obtain from (2.16) and (2.17)

$$
\left\|M_{\varphi_{j}}(f)\right\|_{2, k} \leq C 2^{-j \frac{2 \gamma+d-2}{2}}\|f\|_{2, k} .
$$

Lemma 2.4 There exists a constant $C>0$ such that, for $f \in \mathcal{S}\left(\mathbb{R}^{d}\right)$

$$
\left\|M_{\varphi_{j}}(f)\right\|_{\infty, k} \leq C 2^{j}\|f\|_{\infty, k} \quad j \geq 0 .
$$

Proof. 2.4 From (1.3) and (2.9), one has for $f \in \mathcal{S}\left(\mathbb{R}^{d}\right)$ and $x \in \mathbb{R}^{d}$,

$$
\begin{aligned}
\left|f *_{k} \varphi_{j, r}(x)\right| & \leq \int_{\mathbb{R}^{d}}\left|f(y) \| \tau_{x} \varphi_{j, r}(y)\right| d \nu_{k}(y) \\
& \leq\|f\|_{\infty, k} \int_{\mathbb{R}^{d}}\left|\varphi_{j, r}(y)\right| d \nu_{k}(y) \\
& \leq C 2^{j}\|f\|_{\infty, k} \int_{\mathbb{R}^{d}} \frac{1}{(1+\|x\|)^{2 \gamma+d+1}} d \nu_{k}(y) \\
& \leq C 2^{j}\|f\|_{\infty, k},
\end{aligned}
$$

which gives the result.

Remark 2.1 We observe that for $j=0$ and from Lemmas 2.2, 2.4, we obtain by interpolation (see [14]) that $M_{\varphi_{j}}(f)$ is of strong-type (p,p) with $1<p \leq+\infty$.

Proof of theorem 2.1. According to Remark 2.1, we deduce by interpolation from Lemmas 2.2, 2.3 that

$$
\left\|M_{\varphi_{j}}(f)\right\|_{p, k} \leq C 2^{-j\left(2 \gamma+d-\frac{2 \gamma+d}{p}-1\right)}\|f\|_{p, k},
$$

for $1<p \leq 2$ and $j \geq 0$.

Similarly, according to Remark 2.1, we get by interpolation from Lemmas $2.3,2.4$ that

$$
\left\|M_{\varphi_{j}}(f)\right\|_{p, k} \leq C 2^{-j\left(\frac{2 \gamma+d}{p}-1\right)}\|f\|_{p, k},
$$


for $2 \leq p \leq+\infty$ and $j \geq 0$.

Since for $\frac{2 \gamma+d}{2 \gamma+d-1}<p<\overline{2} \gamma+d$, we have

$$
\sum_{j \geq 0}^{+\infty} 2^{-j\left(2 \gamma+d-\frac{2 \gamma+d}{p}-1\right)}<+\infty \text { and } \sum_{j \geq 0}^{+\infty} 2^{-j\left(\frac{2 \gamma+d}{p}-1\right)}<+\infty .
$$

This yields using (2.8)

$$
\|M(f)\|_{p, k} \leq C\|f\|_{p, k}
$$

which completes the proof.

Remark 2.2 The case $2 \gamma+d=2$ implies that $k \equiv 0$ and was proved by Bourgain in [1].

\section{References}

[1] J. Bourgain, Averages in the plane over convex curves and maximal operators, J. Analyse Math. 47 (1986), 69-85.

[2] F. Dai and H. Wang, A transference theorem for the Dunkl transform and its applications, Journal of Functional Analysis 258 (2010), no. 12, 4052-4074.

[3] C. F. Dunkl, Differential-Difference operators associated to reflextion groups, Trans. Amer. Math. 311 (1989), no. 1, 167-183.

[4] M.F.E. de Jeu, The Dunkl transform, Invent. Math. 113 (1993), no. 1, 147-162.

[5] H. Mejjaoli and K. Trimche, On a mean value property associated with the Dunkl Laplacian operator and applications, Integral Transform. Spec. Funct. 12 (2001), 279-302.

[6] M. Rösler, Generalized Hermite polynomials and the heat equation for Dunkl operators, Comm. Math. Phys. 192 (1998), no. 3, 519-542.

[7] M. Rösler, Positivity of Dunkl's intertwining operator, Duke Math. J. 98 (1999), 445-463. 
[8] M. Rösler, Dunkl operators: theory and applications, Orthogonal polynomials and special functions (Leuven, 2002), Lect. Notes Math. 1817, Springer-Verlag (2003), 93-135.

[9] M. Rösler, Bessel-type signed hypergroup on $\mathbb{R}$, Probability measures on groups and related structures, XI (Oberwolfach, 1994), World Sci. Publ., River Edge, NJ, 1995, pp. 292304.

[10] M. Rösler, A positive radial product formula for the Dunkl kernel, Trans. Amer. Math. Soc. 355 (2003), no. 6, 2413-2438.

[11] M. Rösler, Markov Processes Related With Dunkl Operators, Advances in Applied Mathematics 21, 575-643 (1998).

[12] E.M. Stein, Maximal functions: spherical mean, Proc. Nat. Acad. Sci. U.S.A. 73(1976), 2174-2175.

[13] E.M. Stein, Singular Integrals and Differentiability Properties of Functions, Princeton Mathematical Series 30. Princeton University Press, Princeton, NJ, 1970.

[14] E.M. Stein and G. Weiss, Introduction to Fourier Analysis on Euclidean Spaces, Princeton Mathematical Series 32. Princeton University Press, Princeton, NJ, 1971.

[15] S. Thangavelu and Y. Xu, Convolution operator and maximal function for the Dunkl transform, J. Anal. Math. 97 (2005), 25-56.

[16] A. Zygmund, Trigonometric Series, Vol. I, II. Third edition. Cambridge Mathematical Library. Cambridge University Press, Cambridge, 2002. 